\title{
Rituximab versus cyclophosphamide for the treatment of children with steroid resistance nephrotic syndrome; a clinical trial study
}

\author{
Mohsen Akhavan Sepahi ${ }^{1,2}{ }^{\circledR}$, Najmeh Farahani², Mohammad Reza Razavi ${ }^{1,2^{*}}{ }^{(\mathbb{D}}$, Hossein Heydari ${ }^{2,3}{ }^{(}$, Shahram \\ Arsang-Jang ${ }^{\mathbb{D}}$
}

'Department of Pediatric Nephrology, School of Medicine, Qom University of Medical Sciences, Qom, Iran ${ }^{2}$ Pediatric Clinical Research of Development Center, Hazrat Masoomeh Hospital, Qom University of Medical Sciences, Qom, Iran

${ }^{3}$ Department of Pediatric Infectious disease, School of Medicine, Qom University of Medical Sciences, Qom, Iran ${ }^{4}$ Department of Epidemiology and Biostatistics, Faculty of Health, Qom University of Medical Sciences, Qom, Iran

\section{*Correspondence to Mohammad Reza Razavi, Email: Mrrazavi@muq.ac.ir, Dr.rezarazavimd@Gmail.com}

Received 11 February 2019 Accepted 26 May 2019 Published online 6 July 2019

Keywords: Nephrotic syndrome, Steroid-resistant nephrotic syndrome, Cyclophosphamide, Rituximab

\begin{abstract}
Introduction: The most common complications of the nephrotic syndrome (NS) are the frequent relapses, steroid resistance, and long-term steroid administration.

Objectives: This study aimed to compare the therapeutic effect of rituximab versus cyclophosphamide in the prevention of relapses and the complications of treatment in children with steroid-resistant NS.

Patients and Methods: This clinical trial study was performed on 50 patients with resistant steroidal NS referred to Masoumeh hospital in Qom, Iran. Patients were randomly divided into the two groups including intervention $(n=20)$ and control groups $(n=30)$. In addition to the prednisolone, the intervention group received $375 \mathrm{mg} / \mathrm{m} 2 /$ weekly rituximab intravenously for 4 weeks. The control group received oral doses of cyclophosphamide $2 \mathrm{mg} / \mathrm{kg} / \mathrm{d}$ for 3 months. During treatment, the frequency of relapses, the mean dose of steroid and the complications of treatment were compared with a paired t-test, independent t-test, and chi-square test.

Results: A significant decrease in the mean dose of steroids and the mean number of relapses were seen in patients after administration of rituximab and cyclophosphamide $(P<0.001)$. However, rituximab reduced the dose of steroid administration by $12.25 \mathrm{mg} / \mathrm{d}$, while cyclophosphamide reduced only $2.83 \mathrm{mg} / \mathrm{d}$ $(P<0.001)$. Rituximab reduced the relapse rate two times on average, while cyclophosphamide reduced only 0.5 times $(P<0.001)$. The incidence of complications in the cyclophosphamide group was found to be more severe than the rituximab group.

Conclusion: To compare rituximab versus cyclophosphamide regarding lower the frequency of recurrence after treatment, we found rituximab is a more suitable drug for the treatment of steroid-resistant NS than cyclophosphamide.

Trial Registration: Registration of trial protocol has been approved in Iranian registry of clinical trial (identifier: IRCT20170527034146N2; https://en.irct.ir/trial/37066, \#IR.MUQ.REC.1396.77).
\end{abstract}

\section{Introduction}

Patients with nephrotic syndrome (NS) have significant proteinuria, hypoalbuminemia, edema, and hyperlipidemia $(1,2)$. There may also be symptoms and signs relating to the underlying causes. Normal values for urinary total protein excretion are $<240$ $\mathrm{mg} / \mathrm{m}^{2} /$ day in children, $<6$ months of age, and $<150 \mathrm{mg} / \mathrm{m}^{2} /$ day in older children. NS is defined as leaking of proteins $>40 \mathrm{mg} / \mathrm{m}^{2} /$ hour or $>1 \mathrm{gm} / \mathrm{m}^{2} /$ day in a 24 -hour urine collection or protein creatinine ratio of a spot urine $>2 \mathrm{mg} / \mathrm{mg}$, whereas excretion $>3$ $\mathrm{g} / 1.73 \mathrm{~m}^{2} /$ day is classified as nephrotic range proteinuria in all age groups $(3,4)$.

\section{Key point}

In a clinical trial study on 50 patients with resistant nephrotic syndrome, we found rituximab is a more suitable drug for the treatment of steroid-resistant nephrotic syndrome than cyclophosphamide.

NS occurs in every 1 to 3 cases in 100000 children under the age of 16 years, which, if not treated, has a higher risk of death and infections $(5,6)$. This syndrome is created in all ages, but it is more common in early childhood since the outbreak in children is 15 times that of adults $(6,7)$. The incidence of the disease in the Asian population is

Copyright $\odot 2019$ The Author(s); Published by Nickan Research Institute. This is an open-access article distributed under the terms of the Creative Commons Attribution License (http://creativecommons.org/licenses/by/4.0), which permits unrestricted use, distribution, and reproduction in any medium, provided the original work is properly cited. 
among 9-16 per 100000 children, which occurs twice in girls than boys. NS starts usually between the ages of 2 and 6 years $(5,6)$, while approximately $85 \%$ of patients have minimal change NS $(8,9)$.

In most cases, it is an idiopathic disease, although in some cases, the minimal change NS is secondary to malignancy, drugs, infections, or other factors (2). Clinical manifestations of NS include generalized edema especially in the eyelids in the morning and in the lower limbs during the evening, progressive weight gain and swelling of the abdomen caused by ascites (7).

Children ( 1 to 8 years old) usually well respond to steroid, but some of them may become steroid-dependent or steroid-resistant $(5,6,10)$. Storied-resistant children are being treated with immunosuppressive drugs, which reduce the rate of relapse and long-term renal dysfunction, however, they may increase the complications of the drugs. In the case of cyclosporine or levamisole, leukopenia may exist as compared with steroids (11). Cyclophosphamide and rituximab are administered as an alternative for corticosteroid therapy in the treatment of steroiddependent NS (SDNS), steroid-resistant NS (SRNS), and recurrent NS (9-11). Cyclophosphamide causes prolongation of the duration of remission and reduces the number of relapses; however, several complications may occur, such as hair loss, hemorrhagic cystitis, lymphopenia, neutropenia, and infertility.

Rituximab is a chimeric monoclonal antibody against the protein CD20, which is primarily found on the surface of immune system B cells. When it binds to this protein it triggers apoptosis and cell death of mature and immature B lymphocytes. The relative healing of rituximab will complete after 6 months or 9-12 months of treatment (12).

\section{Objectives}

The objective of this clinical trial is to compare the effects of cyclophosphamide versus rituximab in the treatment of children with SRNS.

\section{Patients and Methods \\ Patients}

Our patients were children with SRNS (entry criteria), who were referred to the Masoumeh hospital in Qom city of Iran (September 2017 to September 2018). Eligible patients were selected according to the criteria of entry and exit of the study by the appropriate method. Accordingly, after explaining the goals of the study, informed consent was obtained from patients or their parents. Then, the eligible patients who agreed to the project were randomly assigned to two groups of intervention and control. Then, the treatment was started after obtaining the approval of the university. Initially, we enrolled 64 children who were divided into two groups of 32 . One group was treated with cyclophosphamide and the other with rituximab. Out of these patients, 12 patients in the rituximab group and 2 patients in the cyclophosphamide group were excluded from the study due to migration and death.

Based on the protocol of the "International Study of Kidney Disease", after the start of treatment with prednisolone $\left(60 \mathrm{mg} / \mathrm{m}^{2} /\right.$ day in 3 divided doses for 4 weeks), we decreased dose of it and after 4 to 6 months, prednisolone was discontinued. In our clinical practice, steroid resistance is defined as inability to induce remission within 4 weeks of daily steroid therapy $(1,6)$.

In the rituximab group were administered $375 \mathrm{mg} /$ $\mathrm{m}^{2} /$ weekly rituximab intravenously for 4 weeks. B cell reduction was monitored using peripheral blood CD19 and CD20 and during the treatment. Additionally, patients' respond to the rituximab, as well complications of rituximab such as infection, fever, nausea, vomiting, low blood pressure, infections or hematological complications were monitored by $\mathrm{CBC}$ differentiation, examination and urinalysis every week.

In the cyclophosphamide group, when there was no response to the steroid, patients were treated orally with $2 \mathrm{mg} / \mathrm{kg} / \mathrm{d}$ cyclophosphamide for 3 months and during the course of treatment. Accordingly, patients' response to the drug and the side effects of cyclophosphamide, such as neutropenia, anemia, lymphopenia, vomiting, thrombocytopenia, urinary cystitis and reproductive effects (not performed in children) were monitored by CBC differentiation examination and urine tests weekly. For both groups in relapse during treatment, the treatment was continued at $60 \mathrm{mg} / \mathrm{m}^{2} /$ day with increasing steroid dose.

The data collection tool was a demographic questionnaire for patients and a checklist in which the age of patients, the age of symptoms, the age at the onset of the symptoms, the age at which the drug was started, gender, the frequency of the relapse of the disease before treatment, the dose of steroids before and after treatment, the regimen status and side effects of each drug were recorded in an information form. The term remission was applied when urinary proteinuria was reduced by $4 \mathrm{mg} / \mathrm{m}^{2} /$ day or less, or the urine strip test was negative or trace (weak band) for 3 consecutive days (Figure 1).

\section{Ethical issues}

This research was conducted following the Declaration of Helsinki principles. Informed written consent was obtained from the patients or their parents. This study was approved by the Ethics Committee of Human Research at Qom University of Medical Sciences (\#IR. MUQ.REC.1396.77, Approval date; 2017-09-05) and registered in Iranian Registry of Clinical Trials (identifier: IRCT20170527034146N2; https://en.irct.ir/trial/37066). This study was extracted from the thesis of Najmeh Farahani in Qom University of Medical Sciences.

\section{Data analysis}

After collecting the data, the descriptive and inferential analysis was performed by SPSS software version 24. Chi- 


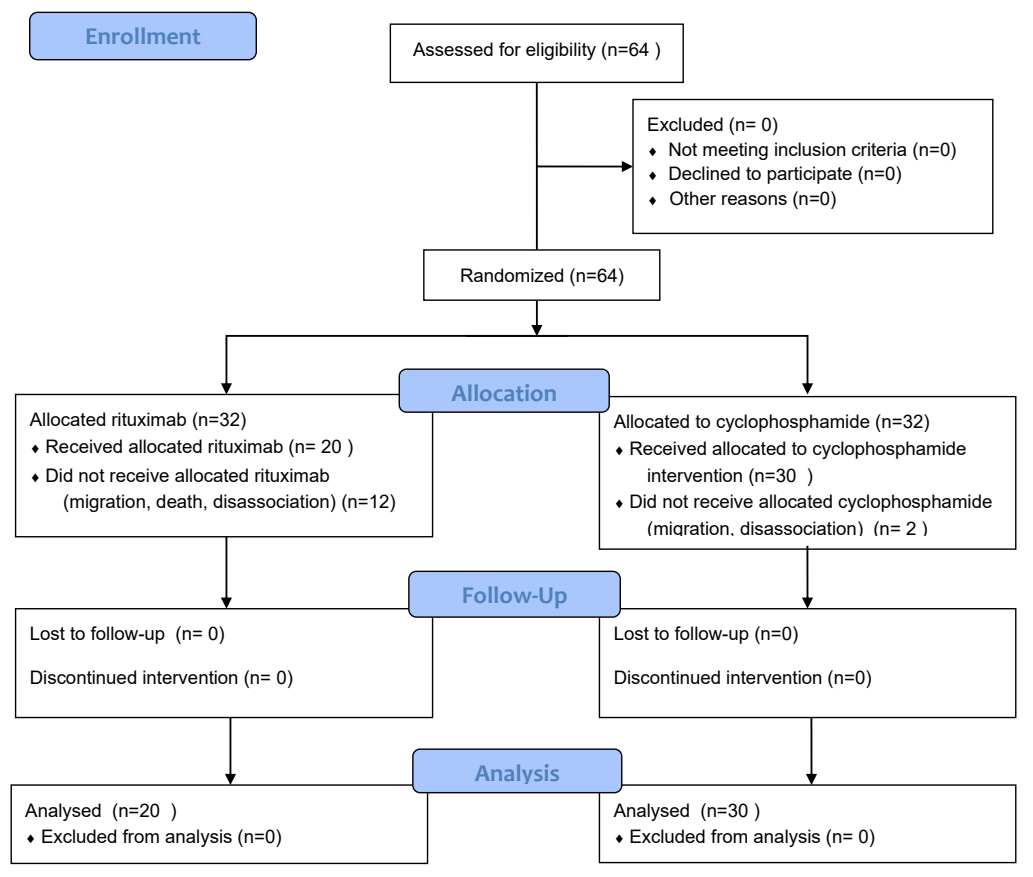

Figure 1. CONSORT Flow diagram of the study.

square test was used for comparing the two groups in terms of qualitative parameters such as gender, the incidence of complications, age of the onset of treatment and age of symptoms. Independent $t$ test was used to compare the amount of steroid used and the relapse rate in two groups. Additionally, paired $t$ test was used to compare the amount of steroid administration and the number of relapses before and after the intervention in each group. Accordingly, $P<0.05$ was considered as statistically significant.

\section{Results}

The age range of the symptoms was between 12 months to 13 years. The mean age of the onset of the symptoms was 4 years. The average age of the onset of rituximab therapy was 7.5 years. The ratio of 12 males $(60 \%)$ and 8 females (40\%) was 3 to 2 .

Table 1 shows the comparison of the intervention rituximab group and the control cyclophosphamide group in terms of gender, age of onset of symptoms and the age of onset of treatment.

Out of 20 children who received the rituximab, 12 patients (60\%) were males and 8 patients (40\%) were females. Out of 30 children who received cyclophosphamide, 21 patients $(70 \%)$ were males and 9 patients (30\%) were females. chi-square test showed no significant differences between these two groups regarding the gender of patients $(P=0.465)$ and the age at the initiation of the treatment $(P=0.239)$.

In the rituximab group, the disease was started at ages between 2 to 6 years old (14 patients, 70\%), and more than 6 years old in 6 patients (70\%).

In the cyclophosphamide group, the age of patients at the initiation of the treatment was between 2 to 6 years old in 16 patients (53.3\%). Additionally 14 patients (46.7\%) were over 6 years of age.

The symptoms were started at the age between 12 months to 13 years old, and the mean age of the onset of symptoms was 4 years.

Table 1. Comparison between the intervention and control groups regarding gender, age at the onset of treatment and symptoms

\begin{tabular}{|c|c|c|c|c|c|c|}
\hline & & \multicolumn{2}{|c|}{ Rituximab } & \multicolumn{2}{|c|}{ Cyclophosphamide } & \multirow{2}{*}{$P$ Value } \\
\hline & & Number & $\%$ & Number & $\%$ & \\
\hline \multirow{2}{*}{ Gender } & Male & 12 & 70 & 21 & 60 & \multirow{2}{*}{0.465} \\
\hline & Female & 8 & 30 & 9 & 40 & \\
\hline \multirow{2}{*}{ Age at the onset of treatment (year) } & $2-6$ & 14 & 70 & 16 & 53.3 & \multirow{2}{*}{0.239} \\
\hline & $>6$ & 6 & 30 & 14 & 46.7 & \\
\hline \multirow{4}{*}{ Age at the onset of symptoms (year) } & 1 & 1 & 5 & 1 & 3.3 & \multirow{4}{*}{0.375} \\
\hline & $1-2$ & 10 & 50 & 10 & 33.3 & \\
\hline & $2-6$ & 7 & 35 & 10 & 33.3 & \\
\hline & $>6$ & 2 & 10 & 9 & 30 & \\
\hline
\end{tabular}


Table 1 shows the incidence of the disease in the rituximab group. Out of 20 patients one was 1 year old (5\%), 10 persons (50\%) were between 1 to 2 years old, 7 persons (35\%) were 2 to 6 years old, while 2 persons (10\%) were more than 6 years old.

In the cyclophosphamide group, out of 30 patients, one person (3.3\%) had one year old, 10 persons (33.3\%) were 1 to 2 years old, 10 patients (33.3\%) were 2 to 6 years old and 9 patients $(30 \%)$ were more than 6 years old. Chi-square test showed no significant differences between the two groups regarding the age of the patients $(P=0.365)$.

Figure 2 shows the incidence of complications in patients taking both drugs. The most important complications were nausea and vomiting, anemia and thrombocytopenia, which occurred in $48.6 \%, 21.4 \%$ and $14.3 \%$ of patients respectively. Chi-square test showed no significant differences between the two groups regarding of incidence of complications $(P=0.328)$.

Although the incidence of infection, nausea and vomiting, thrombocytopenia, neutropenia, and anemia in the cyclophosphamide group were higher than rituximab, it is arguable that in the rituximab group, we had 50\% nausea and vomiting, 15\% thrombocytopenia, 25\% anemia and 5\% for the incidence of lymphopenia, blood pressure, rash, and neutropenia. In the cyclophosphamide group, 7 patients (23.33\%) were diagnosed with thrombocytopenia, 2 patients $(6.67 \%)$ with neutropenia, and one patient (3. 3\%) with exacerbation of underlying disease and decreased glomerular filtration. In cyclophosphamide group, hemorrhagic cystitis was seen in 4 patients (13.3\%), nausea and vomiting in 24 patients (80\%), peritonitis in one patient $(3.3 \%)$ and anemia in 10 patients $(33.33 \%)$. In none of the patients, thrombotic embolism occurred. In the rituximab group, the mean number of the relapses was significantly decreased from $2.90 \pm 0.91$ relapses to $0.9 \pm$ 1.21 times. Additionally, in the cyclophosphamide group, the mean number of relapses decreased significantly from $2.50 \pm 0.86$ incidences to $2 \pm 0.79 \%$.

Paired $t$ test showed that the mean dose of steroids in the rituximab group ranged from $14.25 \pm 2.520 \mathrm{mg} / \mathrm{d}$ before the intervention to $2 \pm 2.38 \mathrm{mg} / \mathrm{d}$ after the intervention. The result shows that the decrease of the steroid dose was statistically significant $(P<0.001)$. The average dose of steroid in the cyclophosphamide group was $13.13 \pm 7.15$ $\mathrm{mg} / \mathrm{d}$ before the intervention to $5.0 \pm 48.5 \mathrm{mg} /$ day after the intervention that was statistically significant $(P=0.019)$ (Table 2). The results of the independent $t$ test showed that the mean of changes in steroid dose in the two groups was significantly different between the groups. In other words, the administration of rituximab reduced an averaged $12.26 \mathrm{mg} / \mathrm{d}$ doses of steroids, while the administration of cyclophosphamide resulted in a reduction of 2.83 doses of steroid in patients that was statistically significant $(P$ $<0.001)$. Accordingly, administration of rituximab resulted in a two-fold decrease in the recurrence of the disease, whereas cyclophosphamide consumption reduced only 0.5 fold in relapse of the disease. This difference was also statistically significant $(P<0.001)$ (Table 3$)$.

In the rituximab group, 20 kidney biopsies were performed that resulted in 8 cases of focal segmental glomerulosclerosis and 12 cases with minor changes. After one year of treatment, relapses occurred in 11 cases (55\%). In 5 cases (25\%) the frequency of relapse was less than the previous year, and in 4 cases (20\%) there were no relapses. In the cyclophosphamide group, 10 kidney biopsies were performed, resulting in segmental glomerulosclerosis in 5 patients and minor changes in 5 patients.

After one year of treatment, remission was seen in two patients $(6.7 \%)$, the frequency of recurrence was reduced to $24 \%(80 \%)$ in 4 patients $(13.3 \%)$, while 2 patients stopped steroid therapy. The amount of steroid consumption reduction before and after the intervention was analyzed in two groups. In the rituximab group, the reduction in steroid consumption was more than 5 times that of the cyclophosphamide group. In addition, the rate of recurrence of the disease before and after intervention in the rituximab group was 4 times more than that of the cyclophosphamide group.

Table 2. Comparison of the mean number of relapses and steroid consumption before and after intervention in two groups

\begin{tabular}{|c|c|c|c|c|c|c|c|c|c|c|}
\hline & & \multicolumn{4}{|c|}{ After intervention } & \multicolumn{5}{|c|}{ Before intervention } \\
\hline & & SD & Mean & Median & Range & SD & Mean & Median & Range & $P$ value \\
\hline \multirow[t]{2}{*}{ Rituximab } & Steroid dose & 2.38 & 2 & 0 & 5 & 5.20 & 14.25 & 15 & 15 & $<0.001$ \\
\hline & Number of relapse & 1.21 & 0.9 & 0 & 3 & 0.91 & 2,90 & 3 & 3 & $<0.001$ \\
\hline \multirow{2}{*}{ Cyclophosphamide } & Steroid dose & 5.48 & 11 & 10 & 20 & 7.15 & 13.83 & 10 & 25 & 0.019 \\
\hline & Number of relapse & 0.79 & 2 & 2 & 4 & 0.86 & 2.5 & 2 & 4 & 0.019 \\
\hline
\end{tabular}

Table 3. Comparison of the mean, median, range in the number of relapses and steroid doses in the patients in the study groups

\begin{tabular}{|c|c|c|c|c|c|c|c|c|c|}
\hline & \multicolumn{4}{|c|}{ Rituximab } & \multicolumn{5}{|c|}{ Cyclophosphamide } \\
\hline & Mean & SD & Median & Range & Mean & SD & Median & Range & $P$ value \\
\hline Steroid dose & -12.25 & 6.53 & -15 & -10 & -2.83 & 6.25 & -15 & -5 & $<0.001$ \\
\hline Number of relapse & -2 & 1.25 & -3 & 0 & -0.5 & 1.11 & 0 & 0 & $<0.001$ \\
\hline
\end{tabular}




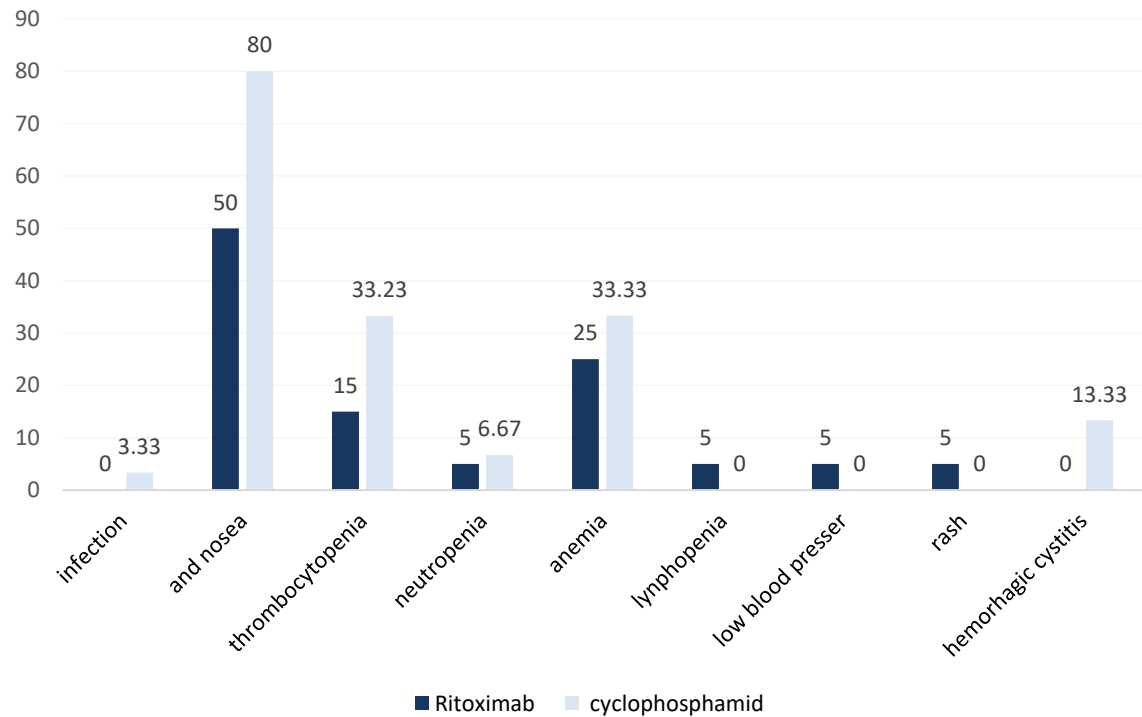

Figure 2. Comparison of relative distribution of drug complications in two study groups.

\section{Discussion}

Several studies have reported the administration of cyclophosphamide versus cyclosporine and levamisole in idiopathic NS $(13,14)$. It was shown that cyclosporine induces more recurrence in patients with SRNS than cyclophosphamide (15). It should be noted that taking cyclophosphamide over 2-3 months maycause azoospermia or infertility in men and may affect the activity of gonads in women $(6,7)$. Treatment with cyclophosphamide can prolong the duration of the remission and reduce the number of relapses, but it may have some complications in the future (16).

The results of this study showed that the frequency of recurrence and the steroid dose were reduced in both groups using rituximab or cyclophosphamide. Based on our results, the mean of steroid dose changes in both groups resulted in an average reduction of $12.25 \mathrm{mg} / \mathrm{d}$ steroid dose. Cyclophosphamide reduced the steroid dose only $2.83 \mathrm{mg} / \mathrm{d}$, while the difference was statistically significant $(P<0.001)$. The effect of rituximab on the reduction of steroid dose was 5 times more than cyclophosphamide and in the prevention of relapse, its effect was 4 times higher $(P<0.001)$.

Other studies have provided similar results in this regard $(17,18)$. Webb et al showed that the effect of rituximab was greater than cyclophosphamide in the treatment of SDNS (19). In 2016, Nakagawa et al showed that rituximab is also effective in SRNS (20). Other studies also showed the effect of rituximab for the treatment of recurrent NS, and in treatment of SDNS $(21,22)$.

In general, when comparing the side effects of rituximab and cyclophosphamide, there is no superiority between them. Although in one study, nausea and skin rash were common in the rituximab group (22). In our study, in the rituximab group, nausea and vomiting (50\%), thrombocytopenia (15\%), anemia (25\%), were seen. In addition, lymphopenia, low blood pressure, rash, and neutropenia were reported as 5\%. Therefore, our study is in line with the study of Webb et al (19) that showed the effect of rituximab treatment was greater than cyclophosphamide for SDNS, since we showed the therapeutic effect of rituximab was greater than cyclophosphamide in the steroid resistance NS.

\section{Conclusion}

In this report, we showed that the use of rituximab in children with SRNS is more effective with less complication. Patients after using rituximab the amount of steroids was reduced by 5 times. However, further studies using a larger sample size are required to confirm the effectiveness of rituximab in SRNS children.

\section{Limitations of the study}

We conducted a single center study with a limited number of patients. Thus, designing larger studies with more participants is suggested.

\section{Conflicts of interest}

The authors declare that they have no competing interest. We did not receive any support from drugs companies, regarding rituximab or cyclophosphamide.

\section{Ethical considerations}

Ethical issues (including plagiarism, double publication) have been completely observed by the authors. This article does not contain any studies with animals performed by any of the authors.

\section{Funding}

This study was extracted from the thesis of Najmeh Farahani in Qom University of Medical Sciences.

\section{References}

1. Ellis D. Pathophysiology, Evaluation, and Management of Edema in Childhood Nephrotic Syndrome. Front Pediatr. 2016;3:111. doi: 10.3389/fped.2015.00111. 
2. Roth KS, Amaker BH, Chan JC. Nephrotic syndrome: pathogenesis and management. Pediatr Rev. 2002;23:237-48.

3. Hoseini R, Sabzian K, Otukesh H, Zafaranloo N, Panahi P, Rahimzadeh N, et al. Efficacy and Safety of Rituximab in Children With Steroid- and Cyclosporine-resistant and Steroidand Cyclosporine-dependent Nephrotic Syndrome. Iran J Kidney Dis. 2018;12:27-32.

4. Eddy AA, Symons JM. Nephrotic syndrome in childhood. Lancet. 2003;362:629-39. doi: 10.1016/S0140-6736(03)14184-0.

5. Dossier C, Lapidus N, Bayer F, Sellier-Leclerc AL, Boyer $\mathrm{O}$, de Pontual L,et al. Epidemiology of idiopathic nephrotic syndrome in children: endemic or epidemic? Pediatr Nephrol. 2016;31:2299-2308. doi: 10.1007/s00467-016-3509-z.

6. Kliegman RM, Lye PS, Bordini BJ, Toth H, Basel D. Nelson Pediatric Symptom-Based Diagnosis E-Book. Elsevier Health Sciences; 2017.

7. Oh W. Nephrology and Fluid/Electrolyte Physiology: Neonatology Questions and Controversies. 3rd ed. Elsevier Health Sciences; 2018.

8. Zhao Z, Liao G, Li Y, Zhou S, Zou H. The efficacy and safety of rituximab in treating childhood refractory nephrotic syndrome: a meta-analysis. Sci Rep. 2015;5:8219. doi: 10.1038/ srep08219.

9. Mohammadjafari H, Nikibakhsh A, Alipour A. The efficacy of rituximab in treatment of childhood steroid resistant and steroid dependent nephrotic syndrome: a systematic review and Meta-analysis. J Pediatr Rev. 2013;1:2-12.

10. Kamei K, Ogura M, Sato M, Sako M, lijima K, Ito S. Risk factors for relapse and long-term outcome in steroid-dependent nephrotic syndrome treated with rituximab. Pediatr Nephrol. 2016;31:89-95. doi: 10.1007/s00467-015-3197-0.

11. Kamei K, Ishikura K, Sako M, Ito S, Nozu K, lijima K. Rituximab therapy for refractory steroid-resistant nephrotic syndrome in children. Pediatr Nephrol. 2018. doi: 10.1007/s00467-0184166-1.

12. Lombel RM, Gipson DS, Hodson EM. Treatment of steroidsensitive nephrotic syndrome: new guidelines from KDIGO. Pediatr Nephrol. 2013; 28:415-26. doi: 10.1007/s00467-012- 2310-x.

13. Madani A, Hajizadeh N, Madani B. Effects of levamisole on children with idiopathic nephrotic syndrome. Feyz Journal of Kashan University of Medical Sciences. 2003;7:16-20.

14. Liu Y, Yang R, Yang C, Dong S, Zhu Y, Zhao M, et al. Cyclophosphamide versus cyclosporine a therapy in steroidresistant nephrotic syndrome: a retrospective study with a mean 5-year follow-up. J Int Med Res. 2018;46: 4506-17. doi: 10.1177/0300060518782017.

15. Rao J, Xu H, Cao Q, Huang WY, Zhou LJ. [Comparison of cyclophosphamide and cyclosporine in the treatment of steroid-resistant idiopathic nephrotic syndrome in children]. Zhong Nan Da Xue Xue Bao Yi Xue Ban. 2007;32:958-63.

16. 16. Honda M. Nephrotic syndrome and mizoribine in children. Pediatr Int. 2002;44:210-6.

17. Gipson DS, Massengill SF, Yao L, Nagaraj S, Smoyer WE, Mahan JD, et al. Management of childhood onset nephrotic syndrome. Pediatrics. 2009;124:747-57. doi: 10.1542/peds.2008-1559.

18. Lombel RM, Gipson DS, Hodson EM. Treatment of steroidsensitive nephrotic syndrome: new guidelines from KDIGO. Pediatr Nephrol. 2013;28:415-26. doi: 10.1007/s00467-0122310-x.

19. Webb H, Jaureguiberry G, Dufek S, Tullus K, Bockenhauer D. Cyclophosphamide and rituximab in frequently relapsing/ steroid-dependent nephrotic syndrome. Pediatr Nephrol. 2016;31:589-94. doi: 10.1007/s00467-015-3245-9.

20. Nakagawa T, Shiratori A, Kawaba Y, Kanda K, Tanaka R. Efficacy of rituximab therapy against intractable steroidresistant nephrotic syndrome. Pediatr Int. 2016;58:1003-1008. doi: 10.1111/ped.12948.

21. Prytuła A, lijima K, Kamei K, Geary D, Gottlich E, Majeed A, et al. Rituximab in refractory nephrotic syndrome. Pediatr Nephrol. 2010;25:461-8. doi: 10.1007/s00467-009-1376-6.

22. Ravani P, Rossi R, Bonanni A, Quinn RR, Sica F, Bodria $M$, et al. Rituximab in Children with Steroid-Dependent Nephrotic Syndrome: A Multicenter, Open-Label, Noninferiority, Randomized Controlled Trial. J Am Soc Nephrol. 2015;26:2259-66. doi: 10.1681/ASN.2014080799. 